\title{
Helicopter Flight Dynamics Modeling and Visual Simulation
}

\author{
Yuan Su ${ }^{1, a)}$, Yuanyuan Huang ${ }^{1, b)}$, Shuai Nie ${ }^{1, c)}$, Jinling Wang², \\ Yihua $\mathrm{Cao}^{1}$, Huanyue Wang ${ }^{1}$ \\ ${ }^{1}$ School of Aerospace Science and Engineering, Beihang University, Beijing 100191, China. \\ ${ }^{2}$ Systems Engineering Research Institute, CSSC, Beijing 100094, China. \\ a)suyuan@buaa.edu.cn \\ b)zy1605126@buaa.edu.cn \\ c)nieshuai@buaa.edu.cn
}

\begin{abstract}
The flight dynamics modeling for helicopter is playing an important role in helicopter design and flight performance analysis. This paper presents aerodynamic models for helicopter components, and the process of constructing aerodynamic model for main rotor is described in detail. When establishing the aerodynamic model for main rotor, a methodology of discretizatin scheme of rotor disk is introduced. Combined all aerodynamic models of helicopter components, taking the gravity and attitude angles into account, the dynamic equilibrium equations of helicopter is firstly derived. Then a comparison in trim calculation was conducted among the SIKOROSKY GEN HEL, the AEFA test and the data from the model established in this paper. The results tending to be consistent validate the accuracy of the model exhibited in this paper. At last, by observing the variation of flight attitudes of helicopter so as to find possible problems in flight process, the visual simulation of helicopter flight process is realized.
\end{abstract}

Key words: Aerodynamic model; Discretization scheme; Flight dynamics modeling; Trim data; Visual simulation.

\section{INTRODUCTION}

Helicopter flight dynamics modeling has been the critical problems in helicopter overall design, flight performance prediction and optimization [1]. The dynamics process of helicopter flight is relatively complex, which contains many nonlinear dynamics problems. The model of nonlinear helicopter flight dynamics is the basis of studying the trim characteristics, stability and controllability of helicopter [2,3]. In recent decades, in order to meet the requirement of the development of high-performance helicopter, related research institutions have made a systematic theoretical study of helicopter flight dynamics model and have developed a variety of helicopter flight dynamics nonlinear mathematical model and improved model for different helicopters. The most representative models are ARMCOP model [4,5] and GEN HEL model [6,7] among them. ARMCOP model takes fully account of the impact, from every components and systems of helicopter, toward the aerodynamics.

\section{HELICOPTER FLIGHT DYNAMICS MODELING}

\section{Aerodynamic model of rotor}

Aerodynamic model of rotor plays a most important role in helicopter flight dynamic model. Set a flying helicopter with velocity $V$ (see Figure 1), the flow condition around blade section is shown in Figure 2. $\alpha, \theta, \varepsilon$ denote blade element angle of attack, pitch angle and inflow angle of attack, respectively. So, $\alpha=\theta+\varepsilon . \beta, \alpha_{S}$ are 
blade flapping angle and disk angle of attack, respectively . $\mu_{T}, \mu_{P}$ is tangential inflow velocity and vertical inflow velocity, respectively. $\psi^{\prime}$ denotes blade azimuth angle. $\Omega$ represents blade rotation angular velocity. $r$ expresses the relative distance from any point of blade to the center of hub. Firstly, the discretization of rotor blade along spanwise direction is shown in Figure 3. Then the rotor disk is divided into $N_{\psi}$ sector regions along azimuth (discrete step length is $\Delta \psi$ ). Therefore, there are $N_{\mathrm{r}} \times N_{\psi}$ grid areas on whole rotor disk shown in Figure 4 . Following this, employing rotor momentum theory and element theory, the aerodynamic model of main rotor is established.

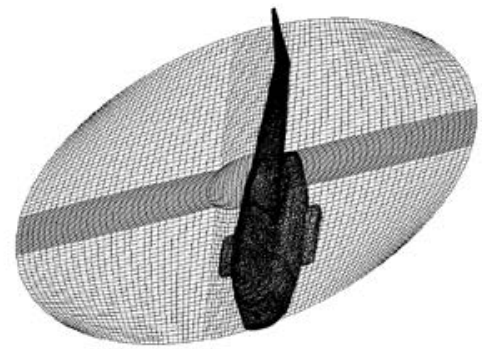

FIGURE 1. Sketch of helicopter and Grid graph

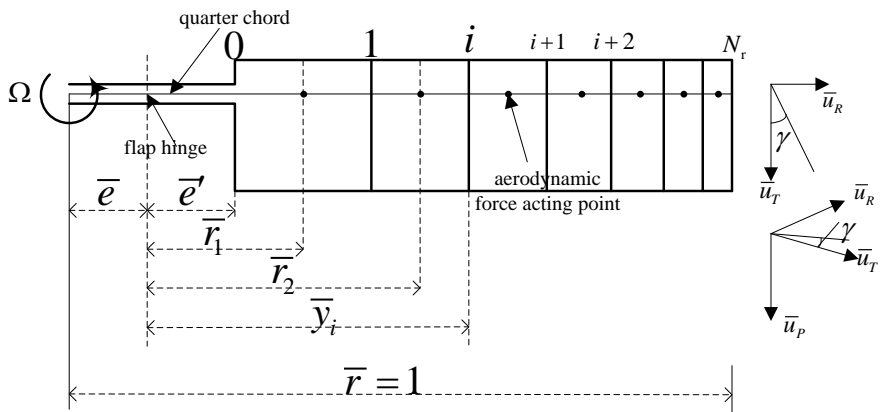

FIGURE 3. discretization of rotor blade along spanwise direction

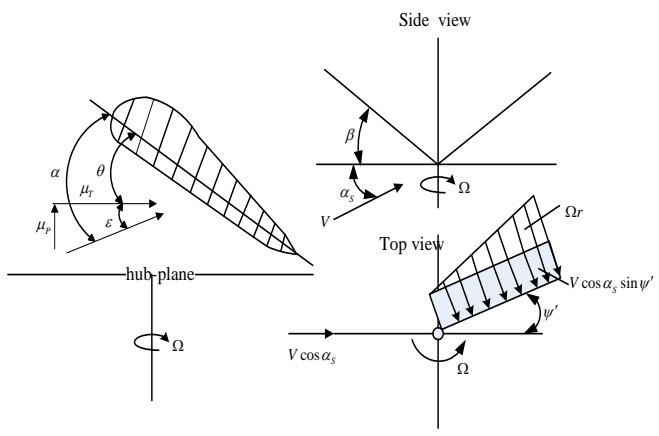

FIGURE 2. Flow condition around blade section.

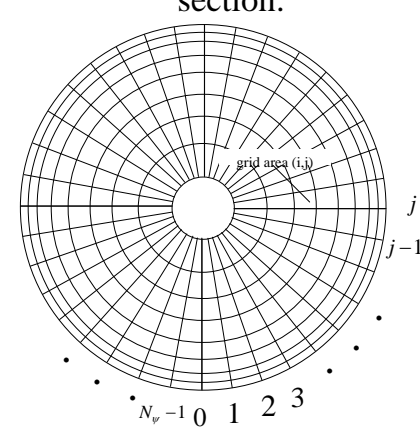

FIGURE 4. schematic drawing of discrezitation of rotor disk $\left(N_{\mathrm{r}}=10, N_{\psi}=36\right)$

\section{Aerodynamic model of fuselage}

Considering the irregularity of fuselage shape, there is not a good theoretical method to simulate the fuselage aerodynamic force. According to numerical wind tunnel data, the aerodynamic force and moment of fuselage is a function of angle of attack and sideslip angle of fuselage. The velocity vector $\left(u_{\mathrm{F}}, v_{\mathrm{F}}, w_{\mathrm{F}}\right)$ can be described as follows:

$$
\left(\begin{array}{c}
u_{\mathrm{F}} \\
v_{\mathrm{F}} \\
w_{\mathrm{F}}
\end{array}\right)=\left(\begin{array}{c}
u_{\mathrm{B}}-q_{\mathrm{B}} \cdot h_{\mathrm{F}, \mathrm{G}}-r_{\mathrm{B}} \cdot d_{\mathrm{F}, \mathrm{G}} \\
v_{\mathrm{B}}+p_{\mathrm{B}} \cdot h_{\mathrm{F}, \mathrm{G}}+r_{\mathrm{B}} \cdot l_{\mathrm{F}, \mathrm{G}} \\
w_{\mathrm{B}}-q_{\mathrm{B}} \cdot l_{\mathrm{F}, \mathrm{G}}+p_{\mathrm{B}} \cdot d_{\mathrm{F}, \mathrm{G}}
\end{array}\right)+\left(\begin{array}{c}
k_{\mathrm{x}, \mathrm{F}} V_{\mathrm{i}} \\
k_{\mathrm{y}, \mathrm{F}} V_{\mathrm{i}} \\
k_{\mathrm{z}, \mathrm{F}} V_{\mathrm{i}}
\end{array}\right)
$$

Where $k_{\mathrm{x}, \mathrm{F}}, k_{\mathrm{y}, \mathrm{F}}, k_{\mathrm{z}, \mathrm{F}}$ denote the rotor-fuselage interference factor, they can be obtained from numerical wind tunnel. Figure 1 depicts the grid graph of helicopter fuselage applied in numerical wind tunnel. 
Then, we can get aerodynamic force $X_{B, F U S}^{\prime}, Y_{B, F U S}^{\prime}, Z_{B, F U S}^{\prime}$ and moment of fuselage $L_{B, F U S}^{\prime}, M_{B, F U S}^{\prime}, N_{B, F U S}^{\prime}$ in body axes system of fuselage. Transformed to helicopter body axes system, they are force $X_{B, F U S}, Y_{B, F U S}, Z_{B, F U S}$ and moment $L_{B, F U S}, M_{B, F U S}, N_{B, F U S}$ of fuselage.

\section{Dynamic model of helicopter}

Synthesizing the aerodynamic model of different parts of helicopter above, the dynamic model of helicopter can be obtained. The force and moment vector of helicopter can be described as follows:

$$
\begin{aligned}
& \left(\begin{array}{c}
X_{\mathrm{B}} \\
Y_{\mathrm{B}} \\
Z_{\mathrm{B}}
\end{array}\right)=\left(\begin{array}{c}
X_{\mathrm{B}, \mathrm{R}} \\
Y_{\mathrm{B}, \mathrm{R}} \\
Z_{\mathrm{B}, \mathrm{R}}
\end{array}\right)+\left(\begin{array}{c}
X_{\mathrm{B}, \mathrm{TR}} \\
Y_{\mathrm{B}, \mathrm{TR}} \\
Z_{\mathrm{B}, \mathrm{TR}}
\end{array}\right)+\left(\begin{array}{c}
X_{\mathrm{B}, \mathrm{FUS}} \\
Y_{\mathrm{B}, \mathrm{FUS}} \\
Z_{\mathrm{B}, \mathrm{FUS}}
\end{array}\right) \\
& \left(\begin{array}{c}
L_{\mathrm{B}} \\
M_{\mathrm{B}} \\
N_{\mathrm{B}}
\end{array}\right)=\left(\begin{array}{c}
L_{\mathrm{B}, \mathrm{R}} \\
M_{\mathrm{B}, \mathrm{R}} \\
N_{\mathrm{B}, \mathrm{R}}
\end{array}\right)+\left(\begin{array}{c}
L_{\mathrm{B}, \mathrm{TR}} \\
M_{\mathrm{B}, \mathrm{TR}} \\
N_{\mathrm{B}, \mathrm{TR}}
\end{array}\right)+\left(\begin{array}{c}
L_{\mathrm{B}, \mathrm{FUS}} \\
M_{\mathrm{B}, \mathrm{FUS}} \\
N_{\mathrm{B}, \mathrm{FUS}}
\end{array}\right)
\end{aligned}
$$

$X_{B, \mathrm{R}}, Y_{B, \mathrm{R}}, Z_{B, \mathrm{R}}$ and $L_{B, \mathrm{R}}, M_{B, \mathrm{R}}, N_{B, \mathrm{R}}$ express force and moment of rotor. $X_{B, \mathrm{TR}}, Y_{B, \mathrm{TR}}, Z_{B, \mathrm{TR}}$ and $L_{B, \mathrm{TR}}, M_{B, \mathrm{TR}}, N_{B, \mathrm{TR}}$ denote force and moment of tail rotor. Taking the gravity of helicopter $m_{\mathrm{G}}$ and attitude angles $\theta, \phi$ into account, the equilibrium equations of six degrees of freedom can be written as follows:

$$
\begin{gathered}
\left(\begin{array}{c}
X_{\mathrm{B}} \\
Y_{\mathrm{B}} \\
Z_{\mathrm{B}}
\end{array}\right)+\left(\begin{array}{ccc}
1 & 0 & 0 \\
0 & \cos \phi & \sin \phi \\
0 & -\sin \phi & \cos \phi
\end{array}\right)\left(\begin{array}{ccc}
\cos \theta & 0 & -\sin \theta \\
0 & 1 & 0 \\
\sin \theta & 0 & \cos \theta
\end{array}\right)\left(\begin{array}{c}
0 \\
0 \\
m_{\mathrm{G}} g
\end{array}\right)=\mathbf{0} \\
\left(\begin{array}{c}
L_{\mathrm{B}} \\
M_{\mathrm{B}} \\
N_{\mathrm{B}}
\end{array}\right)=\mathbf{0}
\end{gathered}
$$

\section{EVALUATION RESULT}

\section{Straight forward flight}

Employing the momentum theory of rotor in descretization scheme, a single rotor helicopter flight dynamic model of UH-60A is developed. As Figure 5 depicts, the trim data calculated with above model, the AEFA TEST[8] , and the SIKORSKY GEN HEL model are consistent with the exception of some discrepancies in the lowspeed flight, which proves that the helicopter model established here is feasible and effective in forward flight. 


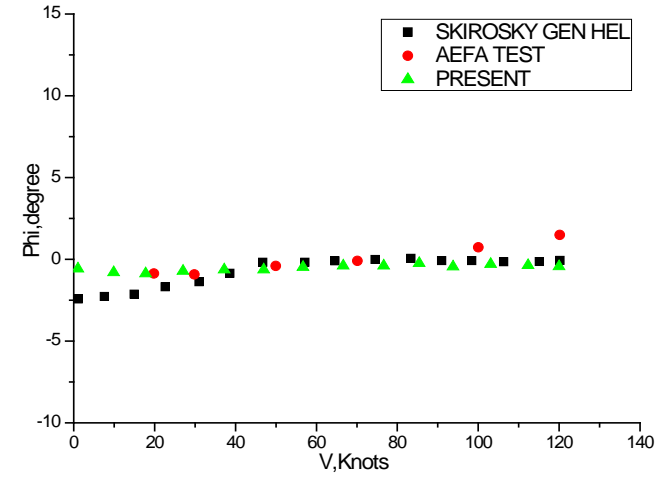

(a) rolling attitude angle

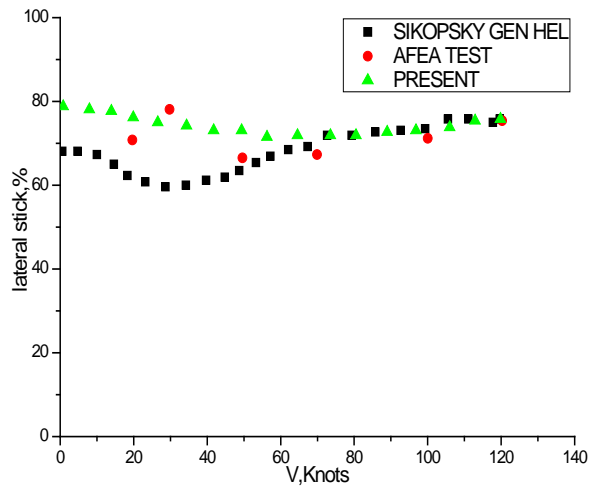

(b) lateral control stick

FIGURE 5. Trim curves and attitude angle in straight forward flight of UH-60A

\section{Climb/glide}

The UH-60A dynamic model based upon blade element theory is used to make trim calculation in flight state of climb and glide at a forward speed of 100 knots. The trim data calculated with the above model has been compared with the SIKOROSKY GEN HEL model, and the AEFA TEST[8] to validate the accuracy of the above model. Figure 6 shows the result of trim calculation in flight state of climb/glide from different models. The computational result was compared with SIKOROSKY data and AEFA TEST data, and good agreement of results validated accuracy of above model.

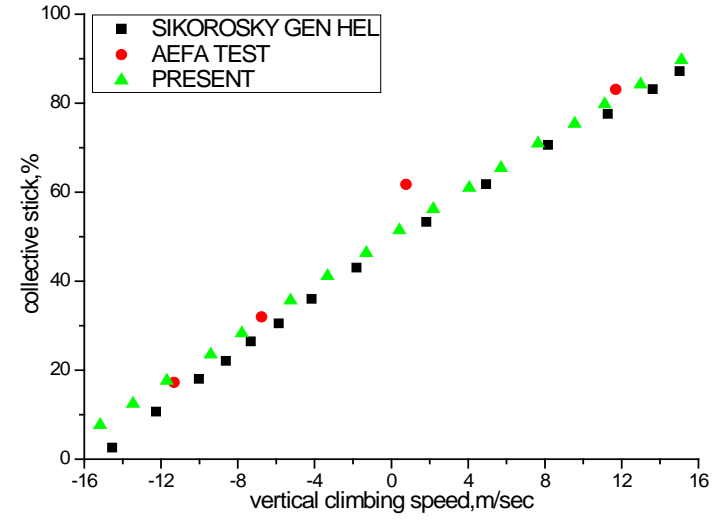

(a) collective control stick

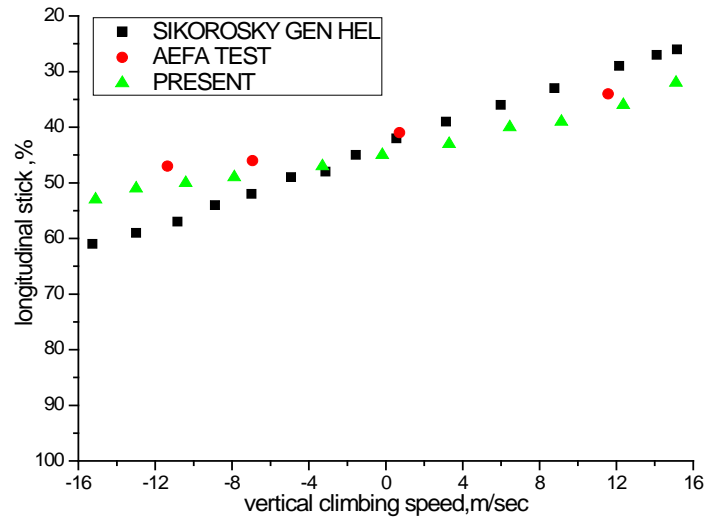

(b) longitudinal control stick

FIGURE 6. Trim curves in climb/glide flight of UH-60A at a forward speed of 100 knots

\section{VISUAL SIMULATION OF HELICOPTER FLIGHT}

After validating the accuracy of flight dynamic model presented in this paper, realizing the visual process of helicopter flight by using visual reality technology $[9,10]$ can help researcher intuitively and effectively observe the flight state of helicopter, including the velocity and attitude. Figure 7 depicts the visual simulation structure diagram. The geometric model of Mi171 helicopter will be chosen to be the simulation object. 


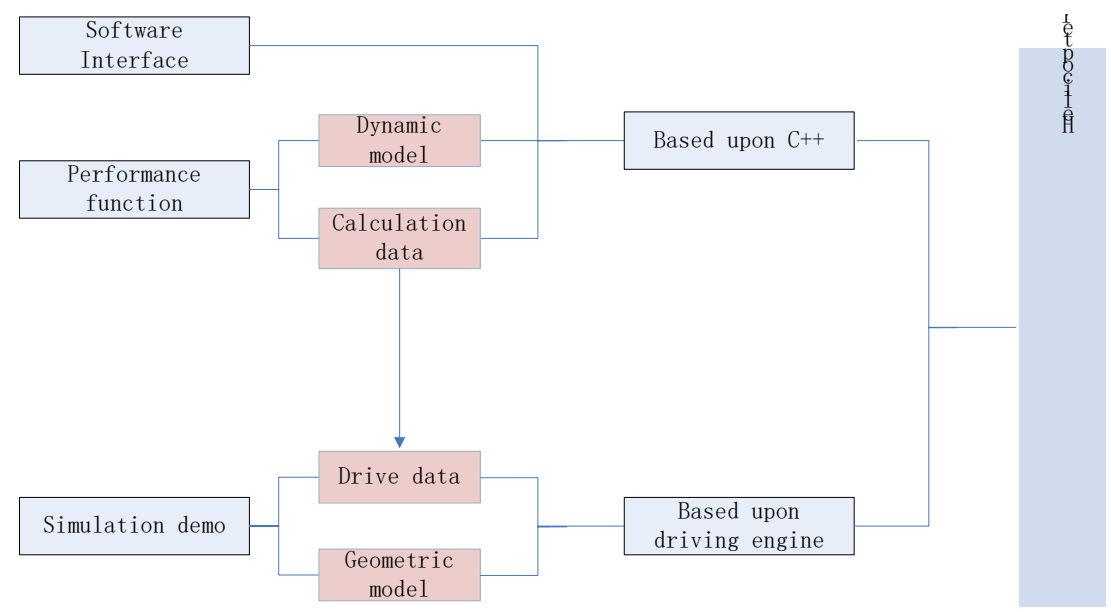

FIGURE 7. Visual simulation structure diagram

\section{Calculation data}

For realizing the visual process of helicopter flight, the attitude of helicopter during flight process is essential. Utilizing the flight dynamic model established above, the attitude angle data of Mi171 in different flight state can also be worked out. The attitude angles of Mi171 for different flight state were shown in Table 1.

TABLE 1. The helicopter attitude in different flight state.

\begin{tabular}{ccc}
\hline Flight State & Pitch Attitude Angle(deg) & Rolling Attitude Angle(deg) \\
\hline Hovering & 0.81324 & -1.25307 \\
Forward Flight & 10.647 & -1.25783 \\
Climb/Glide & -5.4265 & -0.96896 \\
Stedy Coordinated-turn & 2.6348 & 15.39867 \\
\hline
\end{tabular}

\section{Result of exhibition}

Virtual driving engine is the core of virtual reality technology. VTree[11], as an object-oriented graph and image SDK based upon OpenGL[12,13], is chosen to be applied to the helicopter flight visual process in this paper. VTree has its own graphics management structure. Combined the calculation data with the virtual driving engine (VTree), the attitudes of helicopter in different flight state will be exhibited through the flight program based upon $\mathrm{C}++$. Figure 8 shows the attitudes of helicopter in different flight states in visual simulation.

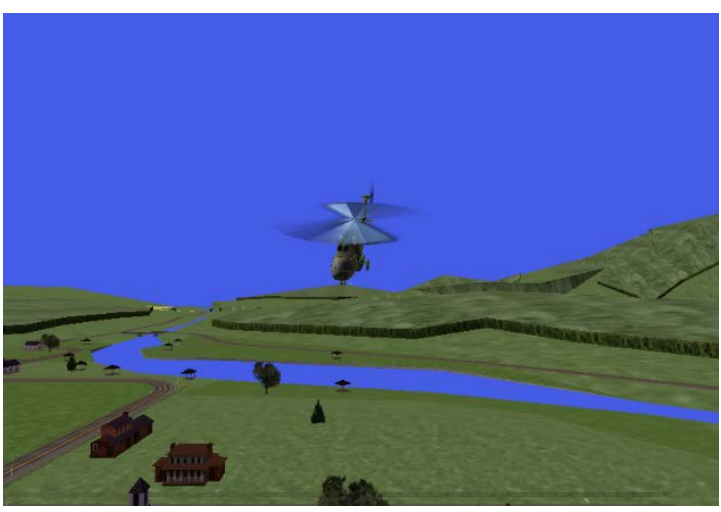

(a)straight forward flight

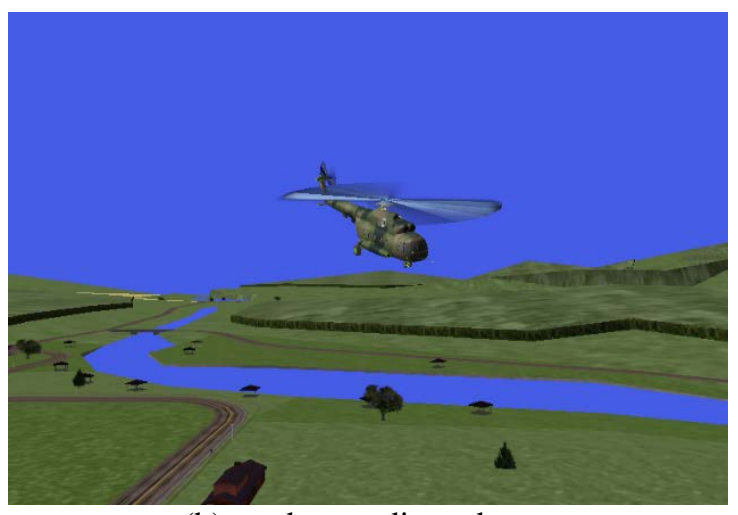

(b)steady coordinated-turn

FIGURE 8. Exhibition of attitudes of helicopter in different flight states 


\section{CONCLUSION}

Based upon the principle of helicopter component modeling, aerodynamic models for each part of helicopter are established separately. A discretization scheme is introduced to establish a discretization model of rotor, including discretization of the rotor blade along the radial direction and generation of structured grids in rotor disk plane. Employing rotor momentum theory and element theory, the aerodynamic model of main rotor is established. Combined with aerodynamic models of other components including tail rotor, horizontal tail and fuselage, formula of the helicopter aerodynamic model is derived. Taking the gravity of helicopter and flight attitudes into account, helicopter flight dynamics equations with six degrees of freedom is thus constructed. Then a comparison among the trim data calculated in this paper, the SIKOROSKY GEN HEL model and AEFA test for different flight states (straight forward flight, climb/glide, steady coordinated-turn) of helicopter UH-60A is conducted. Through the comparison of collective control stick, pedal position, longitudinal control stick, lateral control stick, attitude angle and the rotor torque, the results tending to be in good agreement with exception of some small discrepancies validate the accuracy of the computational model established in this paper. Finally, with the application of virtual reality technology, the helicopter (Mi171) flight process is reappeared. The visual simulation of helicopter flight process can help researchers intuitively observe the variation of attitudes in flight process, therefore it could help them to find possible problem during flight process.

\section{REFERENCES}

1. J.J. Bertin, Aerodynamics for Engineers, fifth ed. Prentice Hall, New Jersey, 1989.

2. J.D. Anderson, Fundamentals of Aerodynamics, third ed., McGraw- HillScience, New York, 2001.

3. R. Gabel, G.J. Wilson, Test approaches to external sling load instabilities, J. American Helicopter Society. 13(1968) 44-55 P.D. Talbot, A mathematical model of a single main rotor helicopter for piloted simulation, R. NASA TM84281, 1982.

4. R.T.N. Chen, A simplified rotor system mathematical model for piloted flight dynamics simulation, R. NASA TM78575, 1979.

5. J.J. Howlett, UH-60A Black Hawk engineering simulation program: Volume I - Mathematical Model, R. NASA CR166309, 1981.

6. M.G. Ballin, Validation of a real-time engineering simulation of the UH-60A helicopter, R. NASA TM88359, 1987.

7. B. Etkin, Dynamics of Atmospheric Flight, first ed., John Wiley \& Sons Inc., New York, 1972.

8. K.B. Hilbert, A mathematical model of UH-60 Helicopter, R. NASATM85890, 1984.

9. G. Burdea, P. Coiffet, Virtual reality technology. Tele operators and Virtual Environments, 12(2003) 663-664.

10. W.R. Sherman, A.B. Craig, Understanding virtual reality-interface, Application, and Design, Tele operators and Virtual Environments 12(2003) 441-442.

11. Z. Xu, S. Tang, X.D. YAN, Application of high fidelity large-scale terrain database in VTree. Computer Simulation 47(2006) 47-50. (IN CHINEASE)

12. D. Shreiner, OpenGL Reference Manual: The Official Reference Document to OpenGL, third ed., AddisonWesley Longman Publishing Co., Boston, 1999.

13. W. Andrew, P. Pierre, F. Alain, A survey of shadow algorithms, IEEE Computer Graphics and Applications 10(1990) 13-32. 